\title{
Molecular Mechanisms and Applications of a Reserpine-Induced Rodent Model
}

\author{
Tayler Hedgecock ${ }^{1}$, Abigail Phillips ${ }^{1}$, Brad Ludrick ${ }^{2}$, Teresa Golden ${ }^{2}$, Ning $\mathbf{W u}^{3 *}$ \\ ${ }^{1}$ Student, Department of Biological Sciences, Southeastern Oklahoma State University, Durant, Oklahoma 74701, USA \\ ${ }^{2}$ Professor, Department of Biological Sciences, Southeastern Oklahoma State University, Durant, Oklahoma 74701, USA \\ ${ }^{3}$ Associate, Professor, Department of Biological Sciences, Southeastern Oklahoma State University, Durant, Oklahoma \\ 74701, USA
}

*Address for Correspondence: Dr. Ning Wu, Associate Professor, Department of Biological Sciences, Southeastern Oklahoma State University, 425 W. University Blvd, Durant, Oklahoma 74701, USA

E-mail: $\underline{\text { nwu@se.edu }}$

Received: 13 June 2018/ Revised: 15 Oct 2018/ Accepted: 18 Dec 2018

\begin{abstract}
Reserpine, an indole alkaloid isolated from the Rauwolfia serpentina has been around since the 1950s. This compound, used for hypertension treatment, had undesirable side effects in patients. The most notable side effect is the induction of depression. Reserpine works by binding irreversibly to the VMAT2 receptor on biogenic amine storage vesicles. This, in turn, causes the storage vesicles to leak their contents into the neuronal cytosol. Cytosolic enzymes such as monoamine oxidases then catabolize and degrade neurotransmitters including Serotonin (5-HT), Dopamine (DA), and Norepinephrine (NE). The depletion of these biogenic amines leads to an increase in depressive-like behavior in various rodents. In addition to the induction of a depressive state, traits associated with pain were also observed in rodent models. Pain is an important characteristic that is co-morbid in patients with major depressive disorder and often referred as the pain-depression dyad. The extent of the induced-depressive state can be determined by several proven tests such as the forced swimming test, open field test, and mechanical allodynia test. Reserpine also induced visible and empirical changes in rodent behavior such as akinesia, ptosis, and hypothermia to name a few. Current researches utilizing reserpine ranging from drug discovery to pathogenesis of diseases such as fibromyalgia are also summarized. Reserpine has been proved the efficacy as a suitable inducer for depression animal model to further the studies at the molecular and systemic levels.
\end{abstract}

Key-words: Animal model, Depression, Molecular mechanism, Neurotransmitter, Reserpine, Rauwolfia serpentina

\section{INTRODUCTION}

Reserpine is a former alkaloid hypertensive drug isolated from the Rauwolfia serpentina, a plant native to an expansive region including India. This indole alkaloid is a diester compound possessing six methoxy groups, nitrogen with base properties and the ability to quaternize and a weak base NH group. Molecular studies have revealed that Reserpine irreversibly binds to the vesicular monoamine transporter 2 proteins (VMAT2), leading to inefficient storage of biogenic amines and causing the neurotransmitters: Serotonin $(5-\mathrm{HT})$,

\section{How to cite this article}

Hedgecock T, Phillips A, Ludrick B, Golden T, Wu N. Molecular Mechanisms and Applications of a Reserpine-Induced Rodent Model. SSR Inst. Int. J. Life. Sci., 2019; 5(1): 2160-2167.

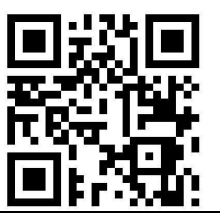

Dopamine (DA), and Norepinephrine (NE) to seep into the cytoplasm ${ }^{[1]}$. As this storage failure progresses, the increase in monoamine concentration in the cytosol of the neurons leads to an increase in Monoamine oxidase (MAO) enzymatic activity. Thus, increasing the catabolism of the vulnerable monoamines leads to their depletion. This molecular action affects the rodent's behavior, and therefore, causes the signs of a depressive-like state, which makes it a potential model to study the molecular mechanism behind depression. Other studies have found a shared co-morbid expression of depression and pain in rodent models, such as C57BL/6 mice, induced by Reserpine ${ }^{[2]}$. Patients suffering from Major Depressive Disorder (MDD) have been recorded to express a lower pain threshold; this is known as the pain-depression dyad ${ }^{[2]}$.

Reserpine's physical appearance is that of a colorless, crystalline powder that has low solubility in water at 
neutral $\mathrm{pH}$ and is generally dissolved using organic acids such as acetic, ascorbic, or citric acids ${ }^{[3]}$. Reserpine, in addition to those organic compounds, can also be dissolved using commercially available solvent mixtures such as benzyl alcohol, polyethylene glycol, and water. In the previous experiment, Minor and Hanff ${ }^{[4]}$ investigated the potential involvement of Reserpine on adenosine signaling leading to the depressive state. Reserpine was dissolved in $10 \%$ dimethyl sulfoxide (DSMO) in physiological saline and administered via intra-peritoneal injection. This variety of chemicals in which Reserpine can be dissolved and administered by allows the compound to possess traction in an experimental setting.

The drug has been around since the 1950's in the medical community, however, its scientific history dates back to the 1930's when its pharmacological capabilities were studied. In "Pharmacology of Reserpine" ${ }^{[5]}$, there were studies already beginning to explore this exotic drug in numerous areas, such as its interactions in the central nervous system (CNS) to lower blood pressure. Throughout the 1950s and early 1960s there was a rise in other studies for Reserpine such as studying its effects on the development of embryos at varying stages, serotonin release during induced convulsions, and then early studies on its effects concerning 5-HT concentrations in rabbit serum ${ }^{[6-8]}$. Within this time period, Reserpine was also found to have no inhibitory effects on acetylcholine, adrenaline, histamine, or noradrenaline, or blocking cellular transmission in autonomic ganglia cells. Weil-Malherbe and Bone studied the effects of Reserpine regarding the change of catecholamine concentration in the brain stem of rabbits and found that the disappearance of biogenic amines occur the most in the supernatant than the mitochondrial fractions ${ }^{[9]}$. The broad effects of Reserpine demonstrate an efficacy for a pharmacologically induced depressive animal model, which can be used for multidisciplinary research activities (Table 1).

\section{Table 1: Potential Applications and Molecular Mechanisms of Reserpine-Induced Animal Models}

\begin{tabular}{|c|c|c|}
\hline Research Application & Molecular Mechanism Relied Upon & References \\
\hline $\begin{array}{l}\text { Alternative Depression Treatment } \\
\text { (Non-Pharmacological) }\end{array}$ & $\begin{array}{l}\text { Utilization of different means of non- } \\
\text { pharmacological treatment for } \\
\text { depression using Reserpine's mode } \\
\text { of action }\end{array}$ & Mohammed $^{[39]}$ \\
\hline $\begin{array}{c}\text { Alzheimer's and Major Depressive } \\
\text { Disorder Pathology }\end{array}$ & $\begin{array}{l}\text { Promotion of inflammatory response } \\
\text { leading to malformed proteins } \\
\text { involved with Alzheimer's Disease }\end{array}$ & Lee et al. ${ }^{[26]}$ \\
\hline Depression-Pain Dyad & $\begin{array}{l}\text { The comorbid expression of pain } \\
\text { pathology through increasing } \\
\text { Substance P levels in addition to } \\
\text { monoamine depletion using } \\
\text { Reserpine }\end{array}$ & $\begin{array}{l}\text { Hanff et al. }{ }^{[1]} \text {, Liu et al. }{ }^{[2]} \text {, Minor and } \\
\text { Hanff }{ }^{[4]} \text {, Freitas et al. }{ }^{[16]} \text {, Ponzio et al. } \\
{ }^{[18]} \text {, Arora and Chopra }{ }^{[22]} \text {, Li et al. }{ }^{[32]} \text {, } \\
\text { Sheppard and Zimmerman }{ }^{[46]}\end{array}$ \\
\hline Drug Discovery & $\begin{array}{l}\text { Utilizing the depletion of } \\
\text { monoamines for a depressive model } \\
\text { that could be reversed by potential } \\
\text { antidepressants }\end{array}$ & $\begin{array}{l}\text { Liu et al. }{ }^{[2]}, \text { Khadrawy et al. }{ }^{[17]} \text {, } \\
\text { Antkiewicz-Michaluk et al. }{ }^{[21]}, \text { Xu et } \\
\text { al. }{ }^{[24]}, \text { Klein et al. }{ }^{[25]}, \text { Gao et al. }{ }^{[31]}, \mathrm{Li} \\
\text { et al. }{ }^{[32]} \text {, Ozerov et al. }{ }^{[35]} \text {, Arora et al. } \\
{ }^{[36]} \text {, Dhir } \text { et al. }{ }^{[37]} \text {, Mahesh et al. }{ }^{[38]} \text {, } \\
\text { Mao et al. }{ }^{[40]}, \text { Mao et al. }{ }^{[41]} \text {, Cui et al. } \\
{ }^{[42]} \text {, Gao et al. }{ }^{[43]} \text {, McGrath et al. }{ }^{[44]} \text {, } \\
\text { Tian et al. }{ }^{[45]}\end{array}$ \\
\hline
\end{tabular}


Fibromyalgia Model

Gene Expression

Irritable Bowel Syndrome Model
Developing a Fibromyalgia model that encompasses the depressionpain expression that is observed in fibromyalgia patients

Examining Reserpine's ability to affect gene expression from its induced oxidative stress and vesicle storage disruption

Developing an IBS model that relies on the addition of the depression exhibited in patients suffering from IBS to study its pathogenesis and
Munro ${ }^{[30]}$, Nagakura et al. ${ }^{[33]}$, Blasco-Serra et al. ${ }^{[34]}$

Vilpoux et al. ${ }^{[19]}$, Urigüen et al. ${ }^{[20]}$, Lee et al. ${ }^{[23]}$, Lee et al. ${ }^{[26]}$

Ghia et al. ${ }^{[27]}$, Minaiyan et al. ${ }^{[28]}$

treatment

Reserpine began to draw attention based upon its ability to affect serotonin levels in the small intestine, and a connection between its ability to affect serotonin and the observable improvements of mental disturbances ${ }^{[10]}$. However, there were five detailed clinical cases of patients being treated with Reserpine for hypertension over a prolonged period developing severe depression ${ }^{[11]}$. In each case, the patients developed the following symptoms including lethargy, unhappiness, withdrawal from the environment, and considering life not worth living. However, anorexia and weight loss did not occur. In addition to the common emotional reaction, two of the patients contemplated suicide. The dosage levels in four of the patients were above the suggested dose limit. However, even when the dose was lowered, the symptoms of induced depressive state persisted until complete withdrawal of Reserpine. The other comparing studies using Losulazine, a hypertensive medication with the similar effects of Reserpine on the depletion of dopamine and norepinephrine, but not as severe, showed that Reserpine is a uniquely potent biogenic amine depleting drug ${ }^{[12]}$.

Molecular mechanisms- Dose-dependency for side effects of Reserpine has been observed in large-scale studies in humans and laboratory experimental animals. Thomas et al. ${ }^{[13]}$ found that there was a trend toward reduced side effects with the lower doses of Reserpine. One of the side effects that was referred in that study was depression with $8.2 \%$ of patients $(n=73)$ at a dosage of Reserpine of $0.25 \mathrm{mg}$ daily. Brodie et al. ${ }^{[14]}$ reported a potential relationship between dose administered and $\mathrm{NE}$ content in the brain stem of rabbits. A dosage as little as $0.1 \mathrm{mg} / \mathrm{kg}$ was effective in lowering norepinephrine content while a dose of $1 \mathrm{mg} / \mathrm{kg}$ was able to deplete $\mathrm{NE}$ content ${ }^{[14]}$.

Reserpine's ability to deplete monoamines was related to its known tranquilizing effect on the specific decrease in 5 -HT levels in rats ${ }^{[15]}$. Freitas et al. ${ }^{[16]}$ examined the dosage effects of Reserpine on mice vacuous chewing movements (VCMs). A negative correlation was observed significantly during the open field test, in which the number of square crossings occurring in a 5 minute period was recorded with the dosage of Reserpine and time elapsed. The control group (after 2 and 20 days) exhibited the most crossed squares, where the number of crossings decreased as the dosage increased from 0.1 to $1 \mathrm{mg} / \mathrm{kg}$. Upon an elapsed time of 60 days, the locomotor activity for the treated mice recovered to that of the control. This is due to the reformation of new monoamine storage vesicles by the cell and a recovery of the biogenic amine concentrations.

Khadrawy et al. ${ }^{[17]}$ suggested that the mechanism of Reserpine is attributed to the fact that it is highly lipid soluble and can penetrate cell membranes. This characteristic of Reserpine explains why in low concentrations over an extended period of time that it can accumulate within cells and bind irreversibly to the protein VMAT2. Ponzio et al. ${ }^{[18]}$ performed an age-based experiment for the effects of Reserpine's mechanism on young and old rats, and the recovery of biogenic amines after Reserpine-induced depletion. Based on the lipid solubility properties of Reserpine, they expected to see a significant difference between the younger, slimmer mice than the older, fatter mice. However, the results demonstrated that a dosage of $5 \mathrm{mg} / \mathrm{kg}$ during their pilot 
experiment was the minimum amount required to affect both groups equally. This pharmacological effect is due to the Reserpine causing depletion of biogenic amines in the same area by binding to VMAT2 irreversibly. The restoration of monoamines was shown to be age independent in this experiment and the mechanism of restoration was not altered.

The effects of Reserpine on the expression of VMAT2 protein were examined to determine if a reduction in serotonin (or enhancement via paroxetine) had effects on the overall gene expression ${ }^{[19]}$. The results of this study found that an acute subcutaneous dosage of 5 $\mathrm{mg} / \mathrm{kg}$ Reserpine did not alter the gene expression, and serotonin level had no effect on the gene expression. The Reserpine model of depression was compared with the olfactory bulbectomy (OB) and chronic corticosterone treatment (CORT) depression models on the basis of gene expression ${ }^{[20]}$. The data from this study showed that the analyzed genes related to DNAdependent apoptosis, regulation of transcription, regulation of programmed cell death, neuron differentiation, negative regulation of transcription, and transcription from RNA polymerase II promoter. However, in comparison to those two animal models of depression, Reserpine animal model showed only two gene expression level changes in common genes shared with the other two models, 55 genes with $\mathrm{OB}$, and 38 genes with CORT. This result illustrates that gene expression level change induced by Reserpine are not the only factor in its depressive-like state, but that it does share some similarities in gene expression with other proven models of depression.

The research performed by Antkiewicz-Michaluk et al. ${ }^{[21]}$ applied chronic low-dosage Reserpine as opposed to a large acute dose. This approach led to a distinct depressive-like behavior (increased immobility time) in rats during the forced swim test. In addition, the chronic Reserpine treatment produced a significant decrease in $\mathrm{DA}, \mathrm{NE}$, and 5-HT levels in the hypothalamus, nucleus accumbens, and ventral tegmental area (VTA). The other study completed by Arora and Chopra observed that the decrease in these neurotransmitters coincided with an increase in the lipid peroxide levels in the cerebral cortex [22]. This heightened concentration of lipid peroxides is caused by the increased action of monoamine oxidases that degrade the biogenic amines affected by Reserpine. The depletion of serotonin in the cerebral cortex leads to an increase in the 5-HT2 receptor on the cell surface, which creates a hypersensitivity effect for serotonin. The neural cells were self-regulated to counteract the low concentration of the neurotransmitters by increasing the sensitivity of the cell to a low concentration of $5-\mathrm{HT}^{[23]}$.

Besides the depressive state, Reserpine can also induce characteristics of pain ${ }^{[24]}$. The pain-depression dyad involves several pathological alterations in the organism. The key changes are the disruption of neuroendocrine and immunologic systems ${ }^{[24]}$. Pain and depression share the same neurotransmitters (5-HT and NE) as well as biological pathways. Although the exact mechanisms are still unknown, this field has been widely studied. Klein, et al. investigated a fibromyalgia model, induced by Reserpine, characterized by chronic widespread pain, fatigue, depression, cognitive dysfunctions, and sleep disturbances [25]. In their research, compounds possessing analgesic effects improved the pain threshold as well as ameliorating depressive behavior, which indicated that the obscure mechanisms shared by pain and depression could be tackled by compounds possessing analgesic and antidepressant properties.

This pharmacological animal model has been shown to share similarities to major depressive disorder (MDD) related diseases on the molecular level in humans. Alterations of gene expression levels for neurotrophic factors and neuronal inflammation have been known in MDD and Alzheimer's disease. The cleavage of the amyloid precursor protein, one of the genetic factors of Alzheimer's disease, was suppressed due to $\gamma$-secretase expression that was inhibited by Reserpine-inducedMDD in mice ${ }^{[26]}$. The Reserpine animal model of depression has been shown to have additive effects for the severity of inflammatory bowel disease (IBD) models, as the concentrations of key inflammatory proteins like IL-1 $\beta$, TNF, and IL- 6 were elevated in the IBD-induced rodents, including an increase in severity of their inflammation ${ }^{[27,28]}$. These pro-inflammatory proteins are also present in the major depressive disorder, illustrating that Reserpine is inducing a depressive-like pathophysiology, which is similar to the other observed human diseases, showing its efficacy of being a suitable human disease animal model.

Observation of Depressive behavior and Experimental model tests- Depression is characterized as changes in mood, sleeping patterns, loss of energy, hopelessness, unexplained aches and pains, changes in appetite, 
unusually slow or fast movements, and/or digestive problems ${ }^{[29]}$. The majority of these changes in behavior can be observed in a controlled laboratory environment. Reserpine has demonstrated ability in inducing many of these behavioral alterations in rodent models. In the conclusion of Munro's study, he pointed out the fact that any of such induced depression experiment should not rely on a single behavior test such as forced swimming test (FST), as this would not be sufficient to solidify a depression model ${ }^{[30]}$. Gao et al. ${ }^{[31]}$ observed that the rats treated with Reserpine traveled the shortest distance in comparison to the control group (saline) in the open field test. Xu et al. ${ }^{[24]}$ examined the depressionpain dyad in mice induced by Reserpine and found a substantial increase in the Substance $P$ levels of the Reserpine treated groups in comparison to that in the control group. In addition, mechanical allodynia was tested and found to have a reduced paw-withdrawal threshold, a test to determine the pain threshold in a model animal, which indicated an exaggerated pain response with depression. This test has been utilized in many experiments using mechanical allodynia as a means to observe the differences in drug treatments $[25,32]$.

The muscle pressure and tactile response thresholds were examined in rats treated with Reserpine. In these experiments male and female rats were used to perform a comparison in a fibromyalgia model, in which depression is a co-morbid symptom for individuals experiencing the disorder ${ }^{[33]}$. In both sexes of rats, the thresholds were drastically reduced in a dose-dependent manner, while the elapsed time between the day one and day seven post injection exhibited the greatest threshold change with $1 \mathrm{mg} / \mathrm{kg}$ dosing. The levels of biogenic amine concentrations in the spinal cord, thalamus, and prefrontal cortex were compared from the day one to seven. However, the concentrations began to recover around the seventh-day post-injection. These results support the facts that Reserpine can induce depression through monoamine depletion accompanied by pain, which allows Reserpine to be a putative method for modeling the depression-pain dyad.

Similar results were found by Blasco-Serra et al. ${ }^{[34]}$, where the pain thresholds in Reserpine treated rats were significantly lowered in comparison to that in the control groups over a seven-day period. In that study, the researchers performed a novelty suppressed feeding test that the rats underwent a food restriction period and were placed in an open field that was illuminated in only the center with a food source. The rats were tested based on how many of them approached the center touched or smelled the food, and the elapsed time for the first feeding event. In each category, the Reserpine treated rats showed the low frequency in approaching the center, inspecting the food, and demonstrated a prolonged period until feeding comparing to that in the control group. Upon being returned to their cages, the rats in both groups had no statistical difference in the time until the first feeding event in the cage or the amount of food consumed. Additional experiment for appetite test to evaluate the degree of the depressivelike state includes sucrose water consumption test, which has also been used to test the effects of antidepressants in increasing the sucrose water consumption by comparing to the induced depression groups without treatment ${ }^{[35]}$.

Another method that is used to test for degree of depression, and commonly used in Reserpine models is the forced swim test (FST). In this test, rodents (rats or mice) are forced to swim in a tank filled with water and the degree of depression is determined by the immobility time of the animal. In comparison to the control, the Reserpine treated animals have drastically prolonged periods of immobility time that signifies a hopelessness behavior in which the animals give up trying to escape from the tank and float ${ }^{[25]}$. The forced swim test has been utilized in several experiments that are screening for potential antidepressant drugs reversing the effect of Reserpine ${ }^{[25,36-39]}$. The tail suspension test (TST) was another proven test for the degree of depression, similar to the FST, the TST tests for the helplessness of the rat and immobility period as it realizes it cannot escape. Just as the FST has been used to screen new antidepressant drugs, the TST can also be utilized in this field ${ }^{[40,41]}$.

Reserpine is also used in testing the frequency of akinesia and ptosis alongside the degree of hypothermia experienced by rodent models. Hypothermia is the condition that the body temperature is lower than normal range. Akinesia is the impairment of the power for one to move voluntarily and results from a decreased level in dopamine that affects the ability of an individual to control the muscles. Ptosis is characterized by the dropping of the upper eyelids and is related to 
depressive-like states. All these physical characteristics can be induced by Reserpine and have been compared amongst control groups for statistical differences in several antidepressant-screening experiments ${ }^{[42-45]}$. The onset of Ptosis was observed to occur at the 2-hour postinjection of Reserpine by Sheppard and Zimmerman ${ }^{[46]}$. The intensity of Reserpine's effects was progressive overtime as ptosis signs to peak at the 8-hour post-administration.

\section{CONCLUSIONS}

Reserpine is an alkaloid indole isolated from the Indian plant Rauwolfia serpentina, which is known to induce depressive-like state in human and animal models. The molecular mechanism involves the irreversible binding to the VMAT2 protein on cellular storage vesicles. This binding causes the vesicles to 'leak' their monoamine contents into the cytosol and exposes them to be degraded by MAO enzymes. This depletion leads to depressive-like symptoms of ptosis, change in appetite, hopelessness, and akinesia to name a few. The degree of the depressive state induced by Reserpine can be determined in controlled laboratory settings by proven tests such as mechanical allodynia (for pain threshold), FST and TST for hopeless- like behavior and monitoring observable ptosis, akinesia, and hypothermia in rodents. Additional research applications using Reserpine induced animal model are being investigated in our laboratory now, which include the studies on Reserpine's potential inflammatory effects that may interact with the neuro-endocrinological systems in depressive animals. With such promised depressive animal model, it provides the effective tools for anti-depressive drug discovery and the development of alternative treatments in the realm of translational medicine.

\section{ACKNOWLEDGMENTS}

This work is supported by the Oklahoma Louis Stokes Alliances for Minority Participation (OK-LSAMP) program.

\section{CONTRIBUTION OF AUTHORS}

Research concept: Ning Wu

Research design: Tayler Hedgecock, Abigail Phillips, Ning $\mathrm{Wu}$

Supervision: Brad Ludrick, Teresa Golden, Ning Wu Materials: Tayler Hedgecock, Abigail Phillips, Ning Wu Data collection: Tayler Hedgecock, Abigail Phillips Data analysis: Tayler Hedgecock, Abigail Phillips, Ning Wu
Literature search: Tayler Hedgecock, Abigail Phillips

Writing article: Tayler Hedgecock, Abigail Phillips, Brad Ludrick, Teresa Golden, Ning Wu

Critical review: Tayler Hedgecock, Abigail Phillips, Brad Ludrick, Teresa Golden, Ning Wu

Article editing: Brad Ludrick, Teresa Golden, Ning Wu

Final approval: Tayler Hedgecock, Abigail Phillips, Brad Ludrick, Teresa Golden, Ning Wu

\section{REFERENCES}

[1] Hanff T, Furst SJ, Minor TR. Biochemical and Anatomical Substrates of Depression and Sickness Behavior. Isr. J. Psychiatry Relat. Sci., 2010; 47(1): 64-71.

[2] Liu S, Zhao R, Li X, Guo H, Tian Z, et al. Attenuation of Reserpine-Induced Pain/Depression Dyad by Gentiopicroside Through Down regulation of GluN2B Receptors in the Amygdala of Mice. Neuromolecular Med., 2014; 16(2): 350-59. DOI: 10.1007/s12017013-8280-8.

[3] Stitzel RE. The Biological Fate of Reserpine. Pharmacol. Rev., 1976; 28(3): 179-208.

[4] Minor TR, Hanff TC. Adenosine signaling in reserpineinduced depression in rats. Behav. Brain Res., 2015; 286: 184-91. DOI: 10.1016/j.bbr.2015.02.032.

[5] Anonymous. Pharmacology of Reserpine. Lancet, 1955; 265(6863): 548-49.

[6] Gal EM, Drewes PA. Levels of Serotonin during Convulsion and the Effect of Reserpine. Nature, 1961; 189: 234.

[7] Limborgh JV, Meenen PV. Effect of Reserpine on Rate of Mortality and Growth of Duck Embryos. Nature, 1963; 197: 615-16.

[8] Naess K, Schanche S. Effect of Reserpine on Serotonin in Rabbit Serum. Nature, 1956; 177: 1130-31.

[9] Weil-Malherbe $H$, Bone AD. Effect of Reserpine on the Intracellular Distribution of Catecholamines in the Brain Stem of the Rabbit. Nature, 1958; 181: 1474-75.

[10]Pletscher A, Shore PA, Brodie BB. Serotonin Release as a Possible Mechanism of Reserpine Action. Science, 1955; 122: 374-75.

[11]Freis ED. Mental Depression in Hypertensive Patients Treated for Long Periods with Large Doses of Reserpine. N Engl J Med, 1954; 251(25): 1006-08.

[12]Pan J, Hooth MJ, Lookingland KJ, Moore KE, Marks TA. Comparison of the Effects of Losulazine and 
Reserpine on Central Aminergic Neurons. Toxicol. Appl. Pharmacol., 1993; 123(1): 26-33.

[13]Thomas et al. Participating Veterans Administration Medical Centers. Low Doses v Standard Dose of Reserpine a Randomized, Double-blind, Multi clinic Trial in Patients Taking Chlorthalidone. JAMA, 1982; 248(19): 2471-77. DOI: 10.1001/jama.1982.03330190035027.

[14]Brodie BB, Olin JS, Kuntzman RG, Shore PA. Possible Interrelationship between Release of Brain Norepinephrine and Serotonin by Reserpine. Science, 1957; 125: 1293-94.

[15]Sulser F, Brodie BB. Is Reserpine Tranquilization Linked to Change in Brain Serotonin or Brain Norepinephrine? Science, 1960; 131: 1440-42.

[16]Freitas CM, Busanello A, Schaffer LF, Peroza LR, Krum $B N$, et al. Behavioral and neurochemical effects induced by reserpine in mice. Psychopharmacology, 2016; 233(3): 457-67.

[17]Khadrawy YA, El-Shamy KA, Mohamed SI. Nicotine restores monoamine neurotransmitter changes in the cortex and hippocampus of reserpinized rats as a model of depression. Eur. Rev. Med. Pharmacol. Sci., 2011; 15(8): 863-70.

[18]Ponzio F, Achilli G, Calderini G, Ferretti P, Perego C, et al. Depletion and recovery of neuronal monoamine storage in rats of different agestreated with reserpine. Neurobiol. Aging, 1984; 5(2): 101-04.

[19]Vilpoux C, Leroux-Nicollet I, Naudon L, RaismanVozari R, Costentin J. Reserpine or chronic paroxetine treatments do not modify the vesicular monoamine transporter 2 expressions in serotonincontaining regions of the rat brain. Neuropharmacology, 2000; 39(6): 1075-82. DOI: 10.1016/s0028-3908(99)00210-5.

[20]Urigüen L, Arteta $D$, Díez-Alarcia R, Ferrer-Alcón $M$, Díaz $A$, et al. Gene expression patterns in brain cortex of three different animal models of depression. Genes Brain Behav., 2008; 7(6): 649-58. DOI: 10.1111/j.1601-183X.2008.00402.x.

[21]Antkiewicz-Michaluk L, Wasik A, Mozdzen E, Romańska I, Michaluk J. Antidepressant-like Effect of Tetrahydroisoquinoline Amines in the Animal Model of Depressive Disorder Induced by Repeated Administration of a Low Dose of Reserpine: Behavioral and Neurochemical Studies in the
Rat. Neurotox Res., 2014; 26(1): 85-98. DOI: 10.1007/s12640-013-9454-8.

[22]Arora V, Chopra K. Possible involvement of oxidonitrosative stress induced neuro-inflammatory cascade and monoaminergic pathway: Underpinning the correlation between nociceptive and depressive behaviour in a rodent model. J. Affect Disord., 2013; 151(3): 1041-52. DOI: 10.1016/j.jad.2013.08.032.

[23]Lee $M$, Wei J. The Influences of Reserpine and Imipramine on the 5-HT2 Receptor Binding Site and Its Coupled Second Messenger in Rat Cerebral Cortex. Chin. J. Physiol., 2013; 56(4): 199-208.

[24]Xu Y, Zhang L, Shao T, Ruan L, Wang L, et al. Ferulic acid increases pain threshold and ameliorates depression-like behaviors in reserpine-treated mice: Behavioral and neurobiological analyses. Metab. Brain Dis., 2013; 28(4): 571-83. DOI: 10.1007/s11011-013-9404-4.

[25]Klein CP, Sperotto ND, Maciel IS, Leite CE, Souza AH, et al. Effects of $D$-series resolvins on behavioral and neurochemical changes in a fibromyalgia-like model in mice. Neuropharm., 2014; 86: 57-66. DOI: 10.1016/j.neuropharm.2014.05.043.

[26]Lee H, Hwang I, Kim J, Choi S, Lee $Y$, et al. Altered expression of $\gamma$-secretase components in animal model of major depressive disorder induced by reserpine administration. Lab Anim. Res., 2012; 28(2): 109-14. DOI: 10.5625/lar.2012.28.2.109.

[27]Ghia J, Blennerhassett P, Collins SM. Impaired parasympathetic function increases susceptibility to inflammatory bowel disease in a mouse model of depression. J. Clin. Invest., 2008; 118(6): 2209-18. DOI: 10.1172/jci32849.

[28]Minaiyan M, Hajhashemi V, Rabbani M, Fattahian E, Mahzouni P. Evaluation of anti-colitic effect of fluvoxamine against acetic acid-induced colitis in normal and reserpinized depressed rats. Eur. J. Pharmacol., 2015; 746: 293-300. DOI: 10.1016/j.ejphar.2014.11.016.

[29]Depression [Internet]. Genetics Home ReferenceNIH U.S. National Library of Medicine. Available from http://ghr.nlm.nih.gov/condition/depression\#statisti cs, 2018; pp. 1-7.

[30]Munro G. Comment on Biogenic amine depletion as a putative animal model of fibromyalgia [Pain 2009; 146: 26-33]. Pain, 2010; 148(1): 172-73. DOI: 10.1016/j.pain.2009.10.018. 


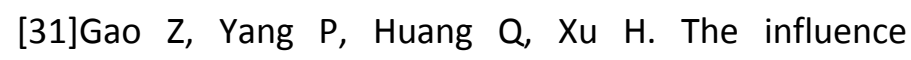
of dizocilpine on the reserpine-induced behavioral and neurobiological changes in rats. Neurosci. Lett., 2016; 614: 89-94. DOI: 10.1016/j.neulet.2016.01.006.

[32]Li S, Han J, Wang DS, Feng B, Deng YT, et al. Echinocystic acid reduces reserpine-induced pain/depression dyad in mice. Metab. Brain Dis., 2016; 31(2): 455-63. DOI: 10.1007/s11011-015-97866.

[33]Nagakura Y, Oe T, Aoki T, Matsuoka N. Biogenic amine depletion causes chronic muscular pain and tactile allodynia accompanied by depression: A putative animal model of fibromyalgia. Pain, 2009; 146(1): 26-33. DOI: 10.1016/j.pain.2009.05.024.

[34]Blasco-Serra A, Escrihuela-Vidal F, Gonzalez-Soler EM, Martínez-Exposito F, Blasco-Ausina MC, et al. Depressive-like symptoms in a reserpine-induced model of fibromyalgia in rats. Physiol Behav., 2015; 151: 456-62. DOI: 10.1016/j.physbeh.2015.07.033.

[35]Ozerov AA, Bagmetova VV, Chernysheva YV, Tyurenkov IN. Comparison of the Efficiency of Adeprophen and Antidepressants of Various Groups on the Model of Reserpine-Induced Depression in Rats. Bull. Exp. Biol. Med., 2016; 160(5): 649-52. DOI: 10.1007/s10517-016-3240-6.

[36]Arora V, Kuhad A, Tiwari V, Chopra K. Curcumin ameliorates reserpine-induced pain-depression dyad: Behavioural, biochemical, neurochemical and molecular evidences. Psychoneuroendocrino., 2011; 36(10): 1570-81.

[37]Dhir A, Malik S, Kessar S, Singh K, Kulkarni S. Evaluation of antidepressant activity of 1-(7methoxy-2-methyl-1,2,3,4-tetrahydro-isoquinolin-4YL)-cyclohexanol, a $\beta$-substituted phenylethylamine in mice. Eur Neuropsychopharmacol., 2011; 21(9): 705-14. DOI: 10.1016/j.euroneuro.2010.12.003.
[38]Mahesh R, Jindal A, Gautam B, Bhatt S, Pandey D. Evaluation of anti-depressant-like activity of linezolid, an oxazolidinone class derivative-An investigation using behavioral tests battery of depression. Biochem. Biophys. Res. Commun., 2011; 409(4): 723-26. DOI: 10.1016/j.bbrc.2011.05.075.

[39]Mohammed HS. Transcranial low-level infrared laser irradiation ameliorates depression induced by reserpine in rats. Lasers Med. Sci., 2016; 31(8): 1651-56.

[40]Mao Q, Huang Z, Ip S, Che C. Antidepressant-like effect of ethanol extract from Paeonia lactiflorain mice. Phytother Res., 2008; 22(11): 1496-99. DOI: 10.1002/ptr.2519.

[41]Mao Q, Ip S, Tsai S, Che C. Antidepressant-like effect of peony glycosides in mice.J. Ethnopharmacol., 2008; 119(2): 272-75. DOI: 10.1016/j.jep.2008. 07.008.

[42]Cui J, Jiang L, Xiang H. Ginsenoside Rb3 exerts antidepressant-like effects in several animal models. J. Psychopharmacol., 2012; 26(5): 697-713. DOI: 10.1177/0269881111415735.

[43]Gao S, Cui Y, Yu C, Wang Q, Zhang Y. Tetrandrine exerts antidepressant-like effects in animal models: Role of brain-derived neurotrophic factor. Behav. Brain Res., 2013; 238: 79-85. DOI: 10.1016/j.bbr. 2012.10.015.

[44]McGrath WR, Ketteler HJ. Potentiation of the Antireserpine Effects of Dihydroxy phenyl alanine by Antidepressants and Stimulants. Nature, 1963; 199: 917-18. DOI: 10.1038/199917a0.

[45]Tian J, Zhang F, Cheng J, Guo S, Liu P, et al. Antidepressant-like activity of adhyperforin, a novel constituent of Hypericum perforatum L. Sci. Rep., 2014; 4: 5632. DOI: 10.1038/srep05632.

[46]Sheppard H, Zimmerman JH. Reserpine and the Levels of Serotonin and Nor-epinephrine in the Brain. Nature, 1960; 185: 40-41. 